\title{
Presumed choroidal naevi and sensory retinal detachment
}

\author{
M. MADISON SLUSHER AND RICHARD G. WEAVER \\ From the Section on Ophthalmology, Department of Surgery, Bowman Gray School of Medicine of \\ Wake Forest University, Winston-Salem, North Carolina
}

SUMMARY Two cases of choroidal naevus in patients with presenting complaints of blurred central vision and subsequent findings of abnormal retinal pigment epithelium (RPE) overlying the pigmented choroidal lesion are presented. Fluoroangiographic evidence of sensory retinal elevation in these areas is provided. Both cases were treated with low-intensity argon laser photocoagulation, with return of central vision without complication. The diagnostic importance of morphologically altered RPE, particularly lipofuscin pigment, overlying such choroidal tumours is stressed. A significant period of follow-up in each case is documented photographically.

Unusual morphological changes in the retinal pigment epithelium (RPE) overlying presumed choroidal naevi may be of significant diagnostic import. Histopathological changes in the retina overlying the naevus and corresponding field defects have been reported as associated findings in such cases (Hale et al., 1965; Flindall and Drance, 1969). Presence of the yellow pigment lipofuscin on the surface of pigmented choroidal lesions has been reported as strongly suggestive of malignant melanoma (Smith and Irvine, 1973). We present two cases in which a choroidal neavus with obvious alterations in the RPE resulted in a sensory retinal elevation which extended into the fovea, causing a reduction in central vision. Argon laser photocoagulation was used to treat a demonstrable fluoroangiographic 'leak', and in both instances it flattened the retina and restored central vision. Follow-up photographs of the lesions at 4 years and 18 months respectively show no discernible change in the naevi.

\section{Case reports}

CASE 1

This 27-year-old White woman presented with a 2-week history of blurred central vision in the right eye. Visual acuity in the right eye was 20/20-3; in the left eye, 20/15. She had metamorphopsia on Amsler grid testing and a corresponding field defect

Address for reprints: Dr M. Madison Slusher, Section on Ophthalmology, Bowman Gray School of Medicine, Winston-Salem, North Carolina 27103, USA. on Goldmann perimetry in the right eye. A large, flat, pigmented lesion with a disturbance in the overlying RPE was observed temporal to the macula in the affected eye (Fig. 1a). Fluorescein angiography confirmed the clinical impression of an overlying serous retinal detachment (Fig. 1c). The clinical appearance of the sensory retinal elevation and visual disturbance remained unchanged for 9 weeks.

The area of leakage was subsequently treated with very low-intensity argon laser photocoagulation. Vision promptly returned to $20 / 15$ and the scotoma disappeared. Four years later the clinical appearance and size of this lesion were unchanged (Fig. 1b).

\section{CASE 2}

A 29-year-old White woman was referred with findings of a flat, dark mass inferior and temporal to the disc in the left eye. The initial symptoms in that eye were visual acuity of $20 / 40$, intense metamorphopsia, and a small field defect on perimetry conforming to the size and location of the lesion (Fig. 2a). The most striking clinical feature of this lesion was the obvious disturbance of the RPE in the form of a yellow pigment deposited on the surface or the choroidal lesion. This disturbance was associated anatomically with areas of 'leak' demonstrated by fluoroangiography (Fig. 2c). Persistence of these findings for 5 months prompted treatment with low-intensity argon laser photocoagulation. Vision improved promptly to $20 / 15$, the subretinal fluid resolved, and her field defect disappeared. An 18-month follow-up fundus photo- 

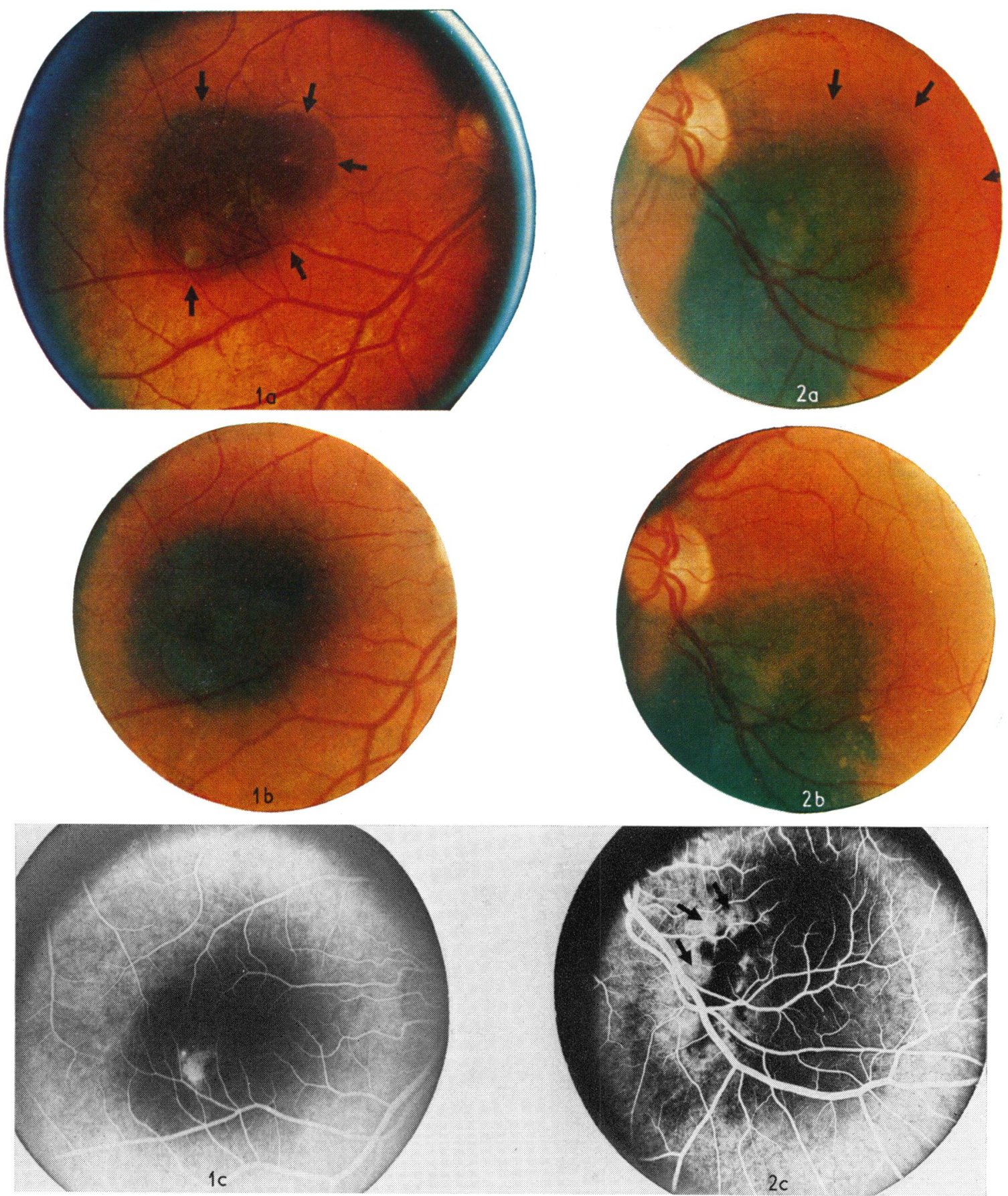

Fig. 1 (a) Fundus photograph of pigmented choroidal mass and RPE disturbance in case I with arrows outlining extent of sensory retinal detachment. (b) This photograph taken 48 months later with different camera. (c)

Morphological disturbance in overlying RPE is seen in central portion of naevus and corresponds to fuorescein leak during venous phase of fluorescein angiography

Fig. 2 (a) Sensory retinal elevation associated with large pigmented choroidal mass, altered RPE, and orange pigment as it presented initially in case 2 . Arrows indicate margins of detached sensory retina. (b) No discernible clinical change in appearance of mass or overlying RPE in 18-month follow-up. (c) Multiple areas of fluorescein leakage occurred around lipofuscin pigment, indicated by arrow's 
graph (Fig. $2 b$ ) indicates no change in the size or appearance of the lesion.

\section{Discussion}

In both patients the presenting complaint of metamorphopsia and blurred central vision led to the discovery of the choroidal lesion. In both cases fluorescein angiography showed the mechanism of visual reduction as a 'leak' in the area of altered RPE (Figs. 1c, 2c). The dense, homogeneous lipofuscin pigment in case 2 represents one type of RPE alteration and characteristically acts as a strong block to choroidal fluorescence. Only very light photocoagulation with an argon laser unit was required to flatten the sensory retina in each patient. In neither case was the clinical appearance of the naevus discernibly altered by the treatment.

With the incidence of choroidal naevi in the general population cited at $9 \%$, Smith and Irvine (1973) have stressed the importance of attempting clinical differentiation between benign choroidal naevi and malignant melanomata. They have shown the importance of orange pigment overlying a choroidal tumour as a helpful criterion in this distinction. Shields et al. (1976) have recently pointed out that lipofuscin pigment is an alteration of RPE cells found in a variety of benign and malignant choroidal tumours, manifesting a differing clinical appearance but demonstrable with fluorescein angiography. The presence of severe RPE abnormalities over any pigmented choroidal lesion justifies a thorough evaluation and long-term follow-up. Both cases presented here, particularly the second case, are being followed up carefully with sequential fundus photographs, visual fields, and fluorescein angiograms.

We gratefully acknowledge the assistance of $\mathrm{Dr}$ J. D. M. Gass in evaluating case 1 , and the kindness of $\mathrm{Dr}$ William Donald for referring case 2. $\mathrm{Mr}$ Marshall Tyler assisted with the photography and fluorescein angiography.

References

Flindall, R. J., and Drance, S. M. (1969). Archives of Ophthalmology, 81, 41.

Hale, P. N., Allen, R. A., and Straatsma, B. R. (1965). Archives of Ophthalmology, 74, 532.

Shields, J. A., Rodriguez, M. M., Sarin, L. K., Tasman, W. S., and Annesley, W. H. (1976). Transactions of the American Academy of Ophthalmology and Otolaryngology, 81, 871.

Smith, L. T., and Irvine, A. R. (1973). American Journal of Ophthalmology, 76, 212. 